\title{
ALGORITHMIC RECONSTRUCTION OF GBM NETWORK COMPLEXITY
}

\author{
Abicumaran Uthamacumaran ${ }^{1}$ and Morgan Craig ${ }^{2,3, *}$ \\ ${ }^{1}$ Department of Physics, Concordia University, Montreal, Canada \\ ${ }^{2}$ Sainte-Justine University Hospital Research Centre, Montreal, Canada \\ ${ }^{3}$ Département de mathématiques et de statistique, Université de Montréal, Montreal, Canada \\ *Corresponding author: morgan.craig@umontreal.ca \\ Lead contact: morgan.craig@umontreal.ca
}




\section{SUMMARY}

Glioblastoma (GBM) is a complex disease that is difficult to treat. Establishing the complex genetic interactions regulating cell fate decisions in GBM can help to shed light on disease aggressivity and improved treatments. Networks and data science offer alternative approaches to classical bioinformatics pipelines to study gene expression patterns from single-cell datasets, helping to distinguish genes associated with control of differentiation and thus aggressivity. Here, we applied a host of data theoretic techniques, including clustering algorithms, Waddington landscape reconstruction, trajectory inference algorithms, and network approaches, to compare gene expression patterns between pediatric and adult GBM, and those of adult glioma-derived stem cells (GSCs) to identify the key molecular regulators of the complex networks driving GBM/GSC and predict their cell fate dynamics. Using these tools, we identified critical genes and transcription factors coordinating cell state transitions from stem-like to mature GBM phenotypes, including eight transcription factors (OLIG1/2, TAZ, GATA2, FOXG1, SOX6, SATB2, YY1) and four signaling genes (ATL3, MTSS1, EMP1, and TPT1) as clinically targetable novel putative function interactions differentiating pediatric and adult GBMs from adult GSCs. Our study provides strong evidence of the applicability of complex systems approaches for reverse-engineering gene networks from patient-derived single-cell datasets and inferring their complex dynamics, bolstering the search for new clinically relevant targets in GBM.

Keywords: Glioblastoma; Complex Systems; Networks; Data Science; Waddington Landscape Reconstruction; Computational Oncology; Pediatric Glioblastoma 
3 Glioblastoma (GBM) is the most lethal pediatric and adult brain tumour. Despite advances in

4 treatment, recurrence will occur in all GBM patients, and mean survival is only 15 months

5 (Alifieris and Trafalis, 2015). GBM is a morbid disease that is driven by a high degree of

6 heterogeneity and phenotypic plasticity in response to the interactions with their tumor

7 microenvironment (Jung et al., 2019). The cell fate transitions and cellular decision-making in

8 GBM cell populations are regulated by the dynamics of complex signaling networks (Suvà et al.,

9 2014; Jia et al., 2017). Recent advances linking single-cell datasets and computational

10 algorithms have improved our understanding of these complex networks and their orchestration

11 of cell fate decisions of GBM transcriptional states (phenotypes) (Jin et al., 2018; lacono et al.,

12 2019). Despite this progress, quantitative approaches that reconstruct the information flow and

13 dynamics of these complex networks remain under-applied. Pediatric GBM exhibits molecular

14 patterns and collective behaviors which are fundamentally different from those of adult GBM

15 (Paugh et al., 2010; Jones et al., 2017; Schwartzentruber et al., 2012; Sturm et al., 2012). There

16 is a greater epigenetic burden in pediatric GBM marked by specific histone H3.3 modifications

17 and aberrant DNA methylation profiles (Scwartzentruber et al., 2012; Sturm et al., 2012; Lulla et

18 al., 2016; Harutyunyan et al., 2019). However, the complex signaling dynamics distinguishing

19 pediatric and adult GBM subgroups, and the similarities within the molecular networks driving

20 their cancer stemness, remain poorly investigated (Paugh et al., 2010; Jones et al., 2017).

21 Answering the question of whether the reconfiguration of these underlying signaling networks in

22 both GBM groups steers their cell fate dynamics would allow for the prediction of causal

23 patterns in the disease progression and therapeutic responses.

25 Glioma-derived stem cells (GSCs) are believed to be a small subset of GBM cancer cells that 26 largely contribute to emergent GBM adaptive behaviors such as phenotypic plasticity, clonal 
27 heterogeneity, self-renewal, aggressiveness (resilience), relapse/recurrence, and therapy

28 resistance (Jung et al., 2019, Xiong et al., 2019). However, many different phenotypes in the

29 tumor microenvironment, including immune cells, healthy cells, extracellular matrices, and blood

30 vessels, form complex feedback loops with malignant GBM cells (Jung et al., 2019, Xiong et al.,

31 2019). GSCs form complex networks with their tumor microenvironment. Signaling dynamics

32 within this microenvironment and its reconfiguration govern the fitness and stemness of GSCs.

33 A lack of quantitative understanding of the causal mechanisms (gene expression patterns)

34 underlying GSC cell fate choices and transitions to their mature phenotypes hinders successful

35 clinical interventions in the treatment of GBM (Jung et al., 2019, Xiong et al., 2019; Yabo et al.,

36 2021).

Statistical approaches are traditionally used to study cell fate dynamics and infer complex networks from large-scale single cell transcriptomics by differential expression analysis through a combination of single cell data processing and clustering algorithms (lacono et al., 2019).

41 However, these algorithmic pipelines are inadequate for capturing the complex patterns and emergent behaviors of cancer network dynamics. Further, fundamental limitations associated with the raw counts of the scRNA-Seq complicate the inference of networks in complex

44 diseases like GBM. These limitations include drop out events (zero counts), and the inherent 45 noise and sparsity of single cell data. To extract quantitatively meaningful differences between

46 GSC and GBM networks, while retaining the essential information representative of their

47 complex dynamics, requires tools from the interdisciplinary paradigm of complex systems

48 theory.

49

50 Complex systems theory, or complexity science, is the study of irreducible systems composed 51 of many interacting parts in which the systems exhibit emergent behaviors. Emergence denotes 52 systems in which the nonlinear interactions between the system and its environment give rise to 
53 complex patterns and unpredicted collective dynamics (Wolfram, 1988; Shalizi, 2006). Some

54 general properties of complex systems include nonlinear dynamics, adaptive processes, self-

55 organized structures, interconnectedness, collective behaviors, pattern formation, fractality,

56 sudden phase-transitions, computational irreducibility, non-locality, long-term unpredictability,

57 undecidability and multi-scaled, multi-nested feedback loops (Wolfram, 1988; Shalizi, 2006).

58 The presence of multi-scaled feedback loops, in particular, is the defining feature of complex

59 networks (Thurner et al., 2018). Traditional reductionist approaches are inadequate to quantify

60 the properties and temporal behaviors of complex networks (Wolfram, 1988; Shalizi, 2006).

61 Complex systems theory advocates the use of computational algorithms and tools from network

62 science to dissect these complex networks (Thurner et al., 2018; Huang et al., 2009; Barabási

63 and Oltvai, 2004).

64

65 The molecular networks coordinating the emergence of GSC and GBM phenotypes are such complex networks. To reveal the mechanisms underlying GSC cell fate decisions and

67 transitions to their mature GBM phenotypes, we deployed several approaches from complex systems theory on data from single-cell RNA Sequencing (scRNA-Seq) count matrices. We compared pediatric GBM to adult GBM to identify the signaling network patterns distinguishing pediatric and adult GBM from GSCs. For this, we relied upon clustering algorithms, Waddington

71 landscape reconstruction, multivariate information theory, network science (graph theory), and

72 machine learning algorithms to map possible cell fate dynamics and identify robust expression

73 markers (critical TFs and genes) driving the complex networks underlying GBM/GSC cell fate

74 control and regulation. We found that distinct gene expression signatures regulate the cell fate

75 decisions in the GBM and GSC patient groups we studied. In particular, we identified a set of

76 key gene targets as master regulators of cell fate decision dynamics in all patient groups, and

77 the critical drivers of GSC stemness networks. Mapping their energy landscape dynamics and

78 cell fate trajectories in pseudotime (cellular transition dimension), we represented the GSC/GBM 
cell fate decisions as dynamical systems which allowed us to identify genes such as GATA2, FOXG1, SATB2, YY1, and SOX6, amidst others, as master regulators of information flow in

81 their signaling networks. Our results help to understand how cellular fate decisions in GBM,

82 identify potential drug targets for precision oncology, and provide a roadmap for data theoretic 83 approaches to other such complex systems.

\section{METHODS}

\section{2.1. General methodological framework}

87 To understand GBM network complexity, we integrated several pediatric and adult IDH-wt GBM single-cell RNA-Seq (scRNA-Seq) datasets in an analytical pipeline that combines several network reconstruction and analysis tools (see subsections below). Details of the datasets used are provided in Table 1. Single-cell datasets were first filtered and normalized in a quality control step, and patient samples were removed from the scRNA-Seq counts expression matrix due to low unique molecular identifier (UMI)/high drop-out rates.

Next, gene expression matrices were analyzed independently using the various clustering and trajectory inference algorithms discussed below. Here we provide a short summary (Figure 1). For the Seurat algorithm, the top 10 principal component analysis (PCA) loadings were used for the differential marker discovery; the top 25 PC loadings were used for the BigScale analysis. To identify the differential markers expressed in all clusters, the top 10 markers within these PC loadings were pooled and analyzed on the UMAP/tSNE patterning space of the cell fate clusters for each patient group. Similarly, the top 2 PCA loadings were used by the scEpath pseudotime analysis. The normalized scRNA-Seq counts of the discovered markers from the Seurat and BigSCale algorithms were pooled together, and separately analyzed for each patient group. The expression counts of these markers were then run through the PIDC Network Inference algorithm to obtain gene receptor networks. 
105 The differential transcription factors identified in the pseudotemporal progression heatmaps

106 were selected for scEpath analysis. Only the markers specific to each patient group were

107 selected for the PIDC network inference. Lastly, complex networks analysis was performed

108 on the reconstructed networks using transitivity and centrality scores to assess the network

109 structure and dynamics (information flow) to identify key regulators of GBM/GSC cell fate

110 decisions. Further, algorithmic complexity measures, as provided in the Supplementary

111 Material, were used to identify gene markers which could accurately discriminate the patient

112 groups by machine learning classifiers. Within the established gene networks, algorithmic

113 complexity was used to identify robust discriminants that could accurately distinguish the

114 three patient groups (i.e., pediatric GBM, adult GBM, and adult GSC), based on the

115 performance of machine learning classifiers on their algorithmic complexity scores (see

116 Supplementary Information). 


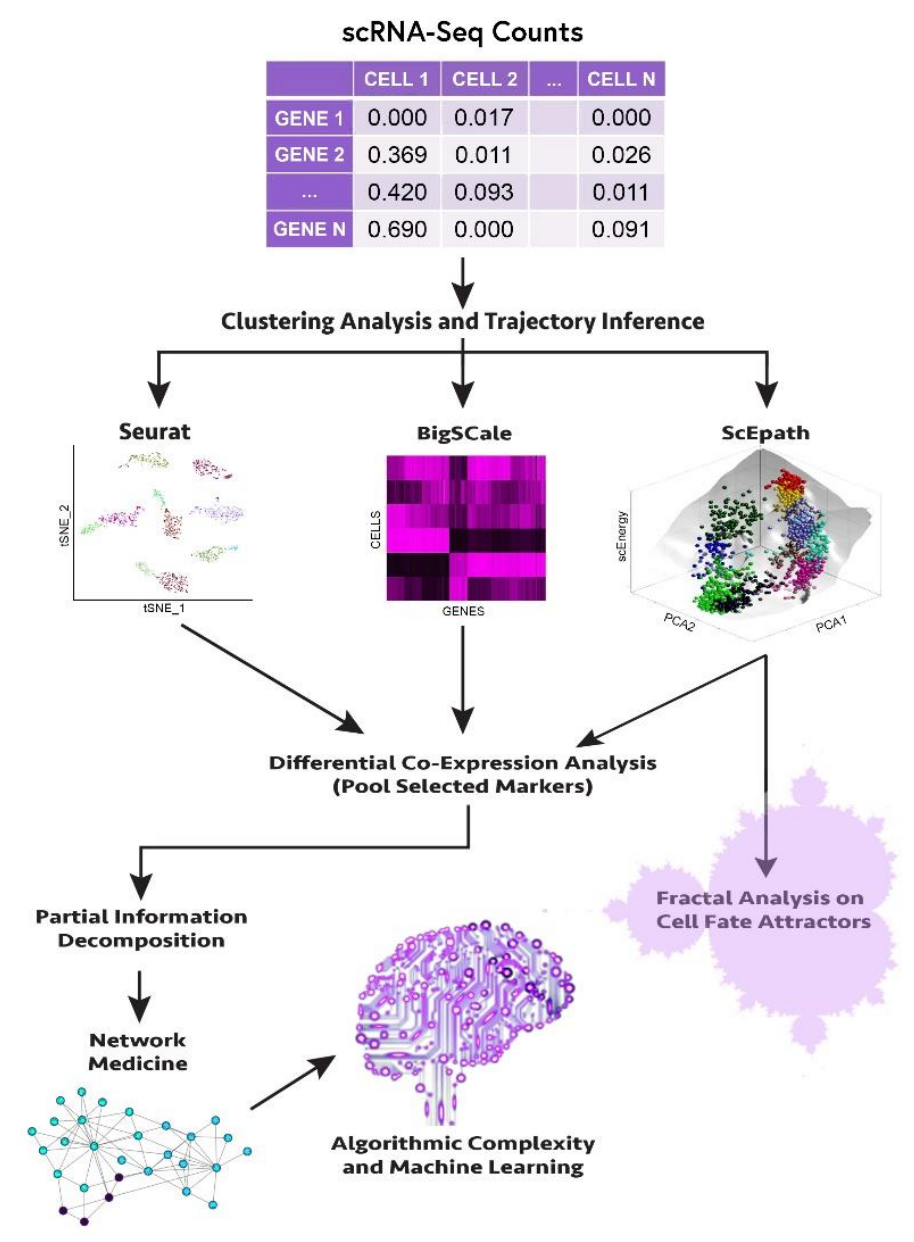

2.2. Single-cell datasets: Gene expression matrices for pediatric GBM, adult GBM, and adult GSC were obtained from the SingleCell Portal repositories from Neftel et al., 2019 and Richards et al., 2021 (Table 1). Briefly, GBM patient samples from Neftel et al. (2019) contained the single cell RNA-Seq counts of four phenotypes (or cellular states): macrophages, malignant GBM cells, oligodendrocytes, and T-cells. Adult GSC consisted only of stem cells. Overall, our dataset included 28 adult GSC datasets, 7 pediatric GBM, and 18 adult GBM scRNA-Seq expression count matrices. 
As a quality control measure for the Seurat and BigSCale clustering, two adult GBM samples and one pediatric GBM sample were dropped in the filtering process (prior to clustering) due to high zero-counts (i.e., low UMI). Importantly, we confirmed that our findings were insensitive to the number of patient samples within each patient group: including these removed samples did not change the differential expression analysis. To further validate this finding, one sample was randomly chosen and dropped from the total number of samples from each patient group to verify whether the clustering analysis changed (i.e., leave-out-one cross-validation) and we confirmed the clustering results were identical. Beyond 2500 cells, the computational time complexity of the scEpath algorithm increased. Thus, the total cell counts of all three patient groups were kept at the maximum computational threshold for the scEpath analysis (see Section 2.3.3). Further, to visualize the cell fate attractor dynamics at the same fine-scale resolution for all patient groups, cell counts were kept roughly the same for each GBM type. Selecting a different combination of adult GSC samples did not change the scEpath landscape or results, as the trial of multiple random selections (> 6 distinct combinations) reproduced identical results. A complete description of the experimental approaches used to derive these datasets from their original studies is provided in the Supplementary Information.

2.3. Clustering techniques. Clustering algorithms were used to identify differential markers co-expressed within all patient groups and distinguish a robust network regulating the cell fate dynamics across all phenotypes.

\begin{tabular}{|l|l|c|c|c|}
\hline $\begin{array}{c}\text { Patient } \\
\text { Group }\end{array}$ & $\begin{array}{c}\text { Single-Cell } \\
\text { Dataset }\end{array}$ & $\begin{array}{c}\text { \# Patient } \\
\text { Samples }(\mathbf{n}) \text { and } \\
\text { Single-Cells (N) } \\
\text { for }\end{array}$ & $\begin{array}{c}\text { \# Patient } \\
\text { Samples (n) and } \\
\text { single-cells (N) } \\
\text { for scEpath } \\
\text { Analysis }\end{array}$ & $\begin{array}{c}\text { \# of Cell Fate } \\
\text { Trajectories in } \\
\text { scEpath } \\
\text { Waddington } \\
\text { Landscape }\end{array}$ \\
\hline $\begin{array}{l}\text { Pediatric } \\
\text { GBM }\end{array}$ & $\begin{array}{l}\text { Neftel et al. } \\
(18)\end{array}$ & $\begin{array}{c}\mathrm{n}=7 \\
\mathrm{~N}=1850\end{array}$ & $\mathrm{n}=7$ \\
$\mathrm{~N}=1850$ & 2 \\
\hline
\end{tabular}




\begin{tabular}{|l|l|c|c|c|}
\hline Adult GBM & $\begin{array}{l}\text { Neftel et al. } \\
(18)\end{array}$ & $\begin{array}{c}\mathrm{n}=18 \\
\mathrm{~N} \sim 21,500\end{array}$ & $\begin{array}{c}\mathrm{n}=7 \\
\mathrm{~N}=2221\end{array}$ & 4 \\
\hline Adult GSC & $\begin{array}{l}\text { Richards et } \\
\text { al. (19) }\end{array}$ & $\mathrm{n}=28$ & $\mathrm{n}=13$ & 2 \\
& $\mathrm{~N} \sim 69,000$ & $\mathrm{~N}=1504$ & \\
\hline
\end{tabular}

TABLE 1. Summary of single-cell datasets. The total number of patient samples $(n)$ and number of single-cells within each patient group $(\mathrm{N})$ used for each step of the clustering and single-cell trajectory inference process are shown.

2.3.1. Seurat algorithm: scRNA-Seq count matrices were pre-processed to obtain normalized and binarized count expressions. Seurat initially performs a cluster analysis by principal component analysis (PCA) dimensionality reduction followed by a graph-based clustering (k-nearest neighbor (kNN) graph) based on the Euclidean distance of the 10 PCA loadings using the FindNeighbors function and Louvain community detection algorithm (modularity optimization) using the FindClusters function (parameter can be tuned between 0.4-1.2 for optimal results), to cluster cells by their Jaccard indexexpression similarity (see Seurat Clustering tutorial in GitHub code). All clustering parameters were kept to their default settings. Next, the cells within the graph-based clusters were visualized on Uniform Manifold Approximation and Projection (UMAP) or tDistributed Stochastic Neighbor Embedding (TSNE) space (i.e., unsupervised nonlinear dimensionality reduction techniques) (Stuart et al., 2019). Differential markers from the top 10 PCA loadings were visualized in UMAP space (analysis does not vary for TSNE space) using the FindAllMarkers function with parameters: min.pct $=0.25$ and logfc.threshold $=0.25$. We clustered similarly expressed cells together in the low dimensional space by finding differentially expressed features/markers corresponding to the highest ten PCA loadings in the graph-based clusters. To identify markers that govern disease progression and transcriptional dynamics, we imposed the condition that selected markers for the network reconstruction must be expressed in all clusters of the three patient groups (pediatric GBM, adult GBM, and adult GSC). 
2.3.2. BigSCale algorithm: BigSCale is a framework for clustering, phenotyping, pseudotiming, and inferring gene regulatory and protein-protein interaction networks from single-cell data (lacono et al., 2019). A SingleCellExperiment class was created from the scRNA-Seq raw count matrices for BigSCale processing, and counts were replaced by z-scores. Cellular clustering was established by first computing all pairwise cell distances using the Pearson correlation to generate a distance matrix. Following, cells were assigned to cluster groups via the Ward's linkage/method (an agglomerative hierarchical clustering algorithm). Iterative differential expression analysis was performed between the clusters of cells and the differential markers within the identified clusters were assessed using the getMarkers function (see BigSCale 2 tutorial in Github

2.3.3. ScEpath algorithm: We applied single cell Energy path (scEpath) to reconstruct the 3Dcode). The markers specific to a cluster were sorted from the highest (most significant) to the lowest (least significant) z-score for the selection of cluster-specific differential and co-expressed gene markers within the top 25 PCA components. A z-score threshold of 5.0 was used as a cut-off threshold while the min_ODscore parameter was kept default at 2.33. This imposed cut-off acts as a filtering mechanism to retain only the markers with significant expression changes per cluster. As in the Seurat analysis, we imposed the condition that selected markers for the network reconstruction must be expressed in all clusters of the three patient groups. energy landscape of cells and infer regulatory relationships from their transcriptional dynamics (Jin et al., 2018). scEpath is a Waddington Landscape reconstruction algorithm with an unsupervised clustering framework for cell lineage hierarchy mapping and studying the pseudotemporal transcriptional dynamics in cell fate decisions. In this trajectory inference algorithm, information flow and network reconfiguration underlying the cellular decision-making steer the topography of cell populations' energy landscapes 
(also referred to as a cell fate landscape, attractor landscape, or Waddington's epigenetic landscape (Waddington, 1957)). A cell state (cell fate) corresponds to a specific transcriptional (gene expression) program and phenotype of a given cellular population. Cell clusters higher on the energy landscape correspond to stem-cell like states (unstable attractors) with higher differentiation potency, while cell states stuck in lower energies (valleys, or stable attractors) correspond to differentiated (mature) phenotypes with lower potency/plasticity (Figure 2).

scEpath allows for the visualization of cell fate transition probabilities in the population, mapping of cell lineage trajectories in pseudotemporal ordering, and inference of cell fate decisions from patient-derived scRNA-Seq datasets using the following steps: (i) preprocessing of scRNA-seq count matrix, (ii) gene regulatory network (GRN) inference, (iii) single cell energy (scEnergy) calculation, (iv) 3D energy landscape reconstruction via principal component analysis and structural clustering; (v) Transition probabilities calculation, (vi) Inference of cell lineage hierarchy via a probabilistic directed graph, (vii) pseudotime trajectory inference and, (viii) downstream analyses of identifying critical transcription factors (TFs) governing the cell-fate commitments (Jin et al., 2018). A detailed description of the scEpath algorithm is provided in the Supplementary Information.

To perform the scEpath analysis on our data, we first pre-processed the log-normalized (within patient-groups) count matrices with respect to their gene expression values by filtering out zero counts. The differential markers were selected from the first two significant PCA components. We then ran the scEpath MATLAB code from (Jin et al, 2018) on these processed datasets. GSC patient samples BT127, BT48, and BT84 from Richards et al. (2021) were used for all scEpath analyses on GSC. Seven pediatric GBM 
samples from (Neftel et al., 2019) and seven adult GBM samples, selected to match the cell count of the pediatric patient group, from (Neftel et al., 2019), were analyzed. We confirmed that the number of patients did not influence the results and analysis by selecting different random sets of adult GBM samples. We then ran energy (Waddington) landscapes reconstruction on the following population sizes: pediatric GBM: $n=7, N=1850$ cells; adult GBM: $n=7, N=2221$ cells; adult GSC: $n=3, N=1504$ cells.

scEpath smooths the average normalized expression of each gene using cubic regression splines to map the pseudotemporal gene expression dynamics along the inferred trajectories of the cell fates on the landscape, leading to smoothed gene expression along a lineage path (Jin et al., 2018). Leveraging this, we inferred key regulatory TFs for the cell fate differentiation by considering all PDG genes with a standard deviation $>0.5$ and a Bonferroni-corrected $p$-value below a significance level $\alpha$ $=0.01$ for the expression greater than a threshold (e.g., log2(fold-change) $>1$ ). The probabilistic-directed graph network and the cell lineage hierarchy inference parameters were kept at default settings (quick_construct $=1$; tau $=0.4$; alpha $=0.01$; theta $1=0.8$ ). The pseudotime-dependent genes were identified using parameters sd_thresh $=0.5$; sig_thresh $=0.01$; nboot (see hyperparameter-optimized code in GitHub link).

2.3.4. Fractal and multifractal analysis: We applied fractal analysis to quantify the complexity of the phenotypic patterns on the scEpath cell fate attractor landscape. Fractals are signatures of complex systems (Mandelbrot, 1982), and the fractal dimension is a non-integer, fractional dimension characterizing the statistical selfsimilarity and roughness of a pattern. A higher fractality in tumor structures may imply that the tumor is more complex, resilient (i.e., withstands environmental perturbations), 
aggressive, and difficult to treat (Coffey, 1998; Baish and Jain, 1998). As such, the

fractal index provides a quantitative measure of the cell fates' phenotypic plasticity (i.e., higher for stem cell-like fates) and disease progression.

We used ImageJ plugin FracLac (v2.5) to compute the fractal dimension (FD) of analyzed samples using the BoxCount algorithm on the cell state attractors (patterns of cellular distributions on the scEpath energy landscapes). To calculate the fractal dimension, landscape images were converted to black and white. Attractor fractal dimensions reconstructed from the cell fate landscapes found to be non-integer were considered to exhibit a fractional dimension in phase-space. Higher fractal indices indicate more complex dynamics that are irregular and asymptotically unpredictable, since in dynamical systems theory, patterns of systems exhibiting deterministic chaos have a fractal dimension (i.e., strange attractors) (Strogatz, 2015). differential expression markers identified by the various approaches discussed above, we reconstructed the underlying complex networks driving the GBM/GSC cell state dynamics on the Waddington energy landscapes. Network inference tools study the statistical dependencies between genes amidst distributions of expression levels in populations of sampled cells (Chan et al., 2017) by inferring a graph-theoretic representation of the functional relationships between the drivers of complex behaviors such as cell fate transitions, thus allowing for the quantification of the relationships between identified differential transition markers and tracking how these relationships change across distinct phenotypes. Partial Information Decomposition and Context (PIDC) networks have been suggested to outperform traditional gene regulatory network inference approaches using correlation metrics, mutual information, Boolean networks, 
or Bayesian inference methods for network reconstruction (Chan et al., 2017). We used this PIDC network inference algorithm to obtain a network structure of GBM and GSC samples.

The Julia packages InformationMeasures.jl and NetworkInference.jl were used to reconstruct the GRN networks. PIDC network inference uses partial information decomposition (PID) to infer regulatory interaction networks from gene expression datasets. We used the NetworkInference.jl package to establish the (undirected) networks from the multivariate information measure (PID) calculated from the gene expression matrices. Gene expression counts were first discretized via Bayesian blocks discretization and the maximum likelihood estimator (Chan et al., 2017). The PIDC network pattern is the simplest network the algorithm can construct such that the distance between the nodes (genes or TFs) are minimized given their weights (PID score). Network measures characterizing the structure, properties, and information flow of these complex networks were then computed and the most differentially expressed genes were identified by the clustering algorithms using PID scores.

2.3.6. Block Decomposition Method Calculations: We evaluated the algorithmic complexity of key nodes (genes) of the inferred signaling networks to further identify robust markers distinguishing GBM and GSC. Algorithmic complexity is a complementary measure that identifies the minimal amount or set of information in our inferred complex networks which regulate the phenotypic plasticity dynamics across the patient groups, and as such the genes/TFs with highest algorithmic complexity could be robust disease screening tools in precision oncology. The K-complexity of a string $s, K(s)$, also known as Kolmogorov or algorithmic complexity, is the shortest computer program length needed to output that string. This can also alternatively be interpreted as the length of 
the shortest description of a system (Zenil et al., 2016). Since $K(s)$ does not depend on a choice of probability distribution like Shannon entropy, it is more robust for the assessment of system complexity (Zenil et al., 2016, Zenil et al., 2019). Formally, the Kolmogorov complexity of a discrete dynamical system is given by

$$
K(s \mid e)=\min \{|p|: U(p, e)=s\}
$$

for a string or array $s$, where $p$ is the program that produces $s$ and halts running on a universal Turing machine $U$ with input $e$. Then, $\mathrm{K}(\mathrm{s})$ is a function that takes a string or matrix $s$ to be the length of the shortest program $p$ that generates s. However, $K(s)$ is in principle incomputable and must be approximated using the coding theorem method (Zenil et al., 2019). We therefore used the Block Decomposition Method (BDM) to approximate the $K(s)$ of a dataset, which provides local estimates of the algorithmic complexity (Zenil et al., 2016). BDM is available in the online algorithmic complexity calculator [OACC] and its R-implementation (see Availability of Data and Material). The BDM is defined as

$$
B D M=\sum_{i=1}^{n} K\left(\text { block }_{i}\right)+\log _{2}\left(\mid \text { block }_{i} \mid\right),
$$

where the block size must be specified for the n-number of blocks. When the block sizes are higher, better approximations of the K-complexity are obtained (Zenil et al., 2016, Zenil et al., 2019). patient samples from each of the three patient groups. String length was kept the same for all gene candidates from each sample. Accordingly, we chose the cell count expressions of 46 cells from each patient sample for this analysis. The R-implementation of the Online Algorithmic Complexity Calculator was used to compute the BDM estimates of K-complexity for each expression string. scNA-Seq counts of the top gene 
interactions with highest PID scores were selected from each network and binarized. Supplementary Information for BDM Results).

\section{RESULTS}

336 3.1. Key driver genes mediating the cell fate transition dynamics in GBM/GSC epigenetic landscapes are identified using the scEpath algorithm.

338 The Waddington landscape reconstruction identified causal patterns (attractors) to which the

339 distinct transcriptional states within each patient group cluster (Fig 2A-C). Distinct patient group

340 clusters were determined by the scEpath algorithm (colored by similarity in gene expression

341 (i.e., phenotypes) in Figure 2). Three and four meta-clusters were identified in the pediatric GBM

342 (Fig 2A) and adult GBM (Fig 2B), respectively while sub-populations are observed within each

343 meta-cluster indicating the presence of phenotypic heterogeneity and epigenetic plasticity. Many

344 genes encoding transcription factors (TFs) were identified as the transition genes required for

345 cells to transition from one attractor to another. We mapped the expressions of these transition

346 genes across the inferred cell fate trajectories (Fig 2D-F) and found similarities in the gene

347 expression signatures and similar oscillatory patterns in EMP1, MTSS1, PHGDH and OLIG1/2

348 (Fig 3). These markers were selected in the clustering and trajectory inference process as

349 explained above. Their similarity was assessed by their expression variation along the cell fate

350 trajectories in pseudotime (Figure 3). We also identified OLIG1/2 as critical transcription factors

351 in the adult GSC phenotypic transitions (Fig 2F). 

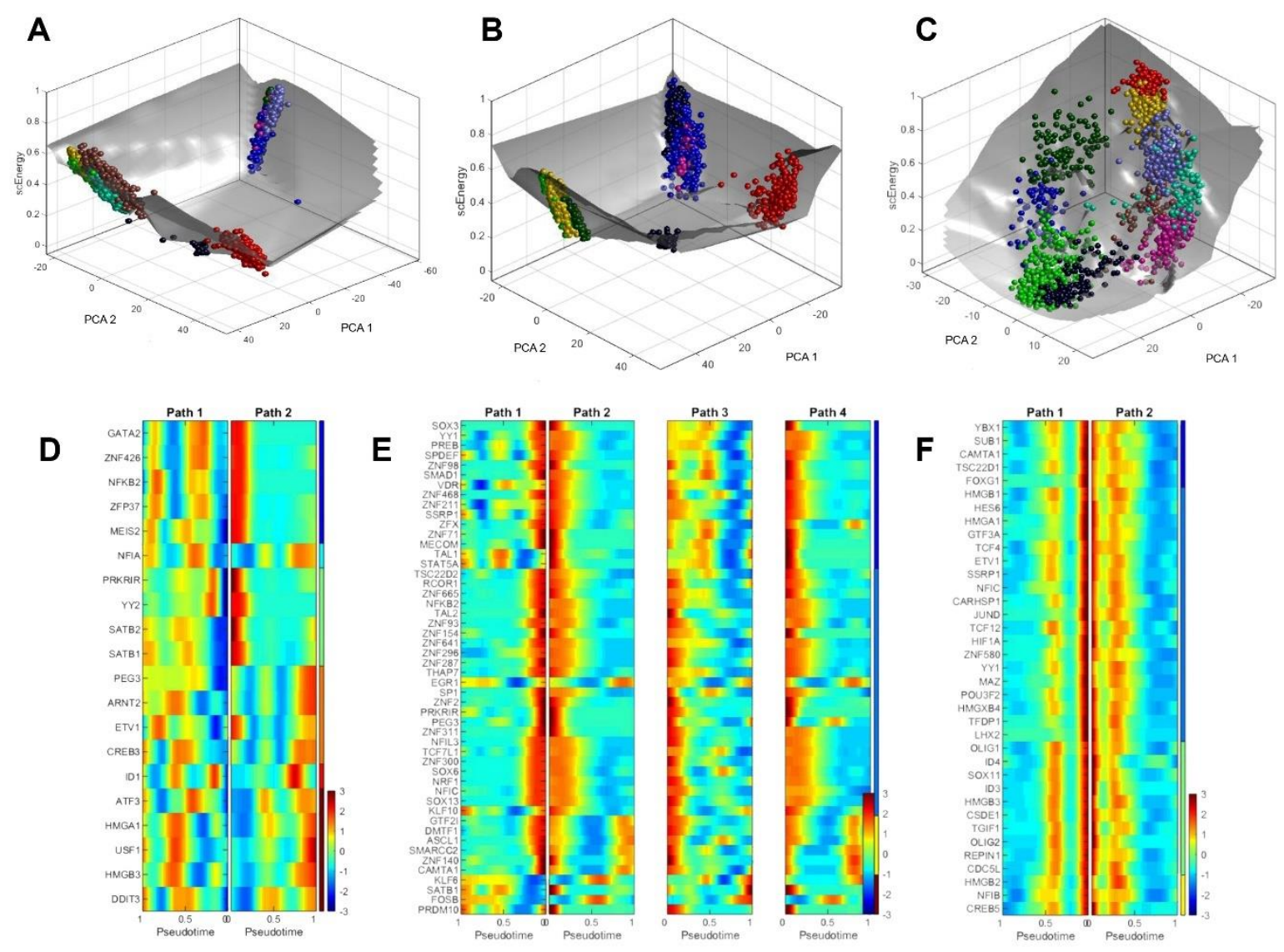

FIGURE 2. Waddington landscape reconstruction differentiates adult and pediatric GBM, and adult GSC critical genes and transcription factors for cell fate transitions. A-C) The distinct phenotypes of each patient group were clustered on the Waddington energy landscape by their similarity in gene expression. The cell clustering patterns on the landscape are referred to as attractors. Balls represent the transcriptional states (cell fates) and paths correspond to the cell fate differentiation trajectories on the Waddington landscape. A) Pediatric GBM. B) Adult GBM. C) Adult GSC. D-F) Heat maps of the critical transcription factors involved in the differentiation and cell fate transitions between the distinct attractors (phenotype clusters) of the GBM/GSC Waddington landscape. The color gradient represents the intensity of the gene expression in pseudotime trajectory, where blue implies low expression and red implies high expression of the gene (TF) during the cell fate choices along the cell differentiation trajectories. The path corresponds to the inferred trajectories in between the cell state attractors on the 


\subsection{Pseudotime expression dynamics identifies oscillatory patterns in critical gene}

\section{targets.}

371 Given the key driver genes and transcription factors identified by scEpath trajectory inference,

372 we next sought to infer similarities in gene expression dynamics during cell fate transitions

373 within each patient group amidst the identified critical gene markers. Using clustering algorithms

374 (see Methods), we found that PTPRZ1 and S100B showed nearly identical expression

375 dynamics in pediatric GBM along both cell fate trajectories on the Waddington landscape,

376 whereas genes such as EMP1, MTSS1, and PHGDH had more complex dynamics and

377 exhibited oscillations during cell fate dynamics in pediatric GBM and adult GSC (Fig 3A and Fig

$3783 \mathrm{C})$. The expression metric used to compare the dynamics of the different pseudotime-

379 dependent genes correspond to the cubic spline smoothened average normalized expression

380 along the pseudotime interval of $[0,1]$.

In adult GBM, NACA and PABPC1, and TPT1 and PSAP had similar expression patterns across all four differentiation paths (Fig 3B). S100B, OLIG1, and PHGDH all had a broad expression

384 profile in path 4 (Fig 3B). Furthermore, the presence of four cell clusters in adult GBM

385 landscape (Fig 2B) is in good agreement with previous classifications of four molecular

386 subtypes of adult GBM (Verhaak et al., 2010). The expression of EGFR and PDGFRA were

387 distinctly higher in one of the four cell fate clusters/attractors (Figure S4B). However, the

388 expression of IDH1 exhibited oscillatory dynamics in all four paths/attractors (data not shown).

389 In adult GSC, many of the identified markers had similar gene expression profiles in

390 pseudotemporal ordering (Fig 3C). For instance, PTPRZ1, NACA and PABPC1, were all found

391 to have similar expression dynamics in both transition paths (Fig 3C). Notably, OLIG 1 and

392 OLIG2 were found to have similar expression patterns in all three patient groups across all cell

393 fate transition trajectories of the landscape (Fig 3A-C). 
395 Notably, we identified that genes such as STMN3, MTSS1 and TAZ are critical regulators in one

396 transition pathway, while PSAP, TPT1, and PTPRZ1 are relevant for the other transition

397 trajectory on the pediatric GBM's Waddington landscape (Fig 3A and S4A). The same trends in

398 pseudotemporal gene expression patterns in STMN3 and PTPRZ1 have also been found in the

399 adult GSC cell fate trajectories (see Fig S4C in the Supplementary Information). In all three

400 patient groups, OLIG1, OLIG2, PHGDH, and TIMELESS had similar expression profiles within

401 the distinct cell fate transition paths indicating potentially some network coordination or

402 collective oscillations. Some signals (e.g., BCAN and CLU) were found to exhibit oscillations

403 that may be indicative of complex dynamics with time-series expression analysis

404 (Supplementary Information). These findings suggest that the identified markers involved in

405 GBM/GSC cell fate decisions exhibit similar patterns in their expression dynamics, and that the

406 identified critical genes are functionally putative master orchestrators of cell fate

407 transitions/differentiation of the heterogeneous phenotypes within a GBM patient's tumor. 


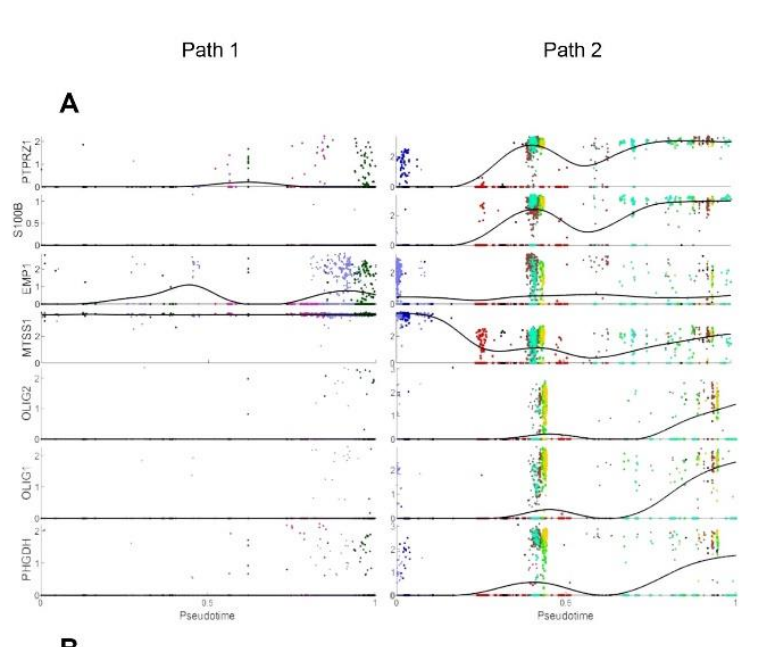

B

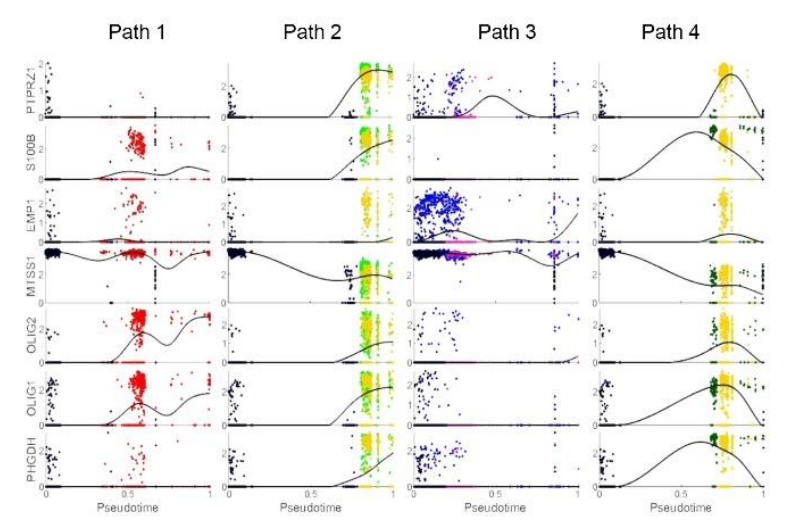

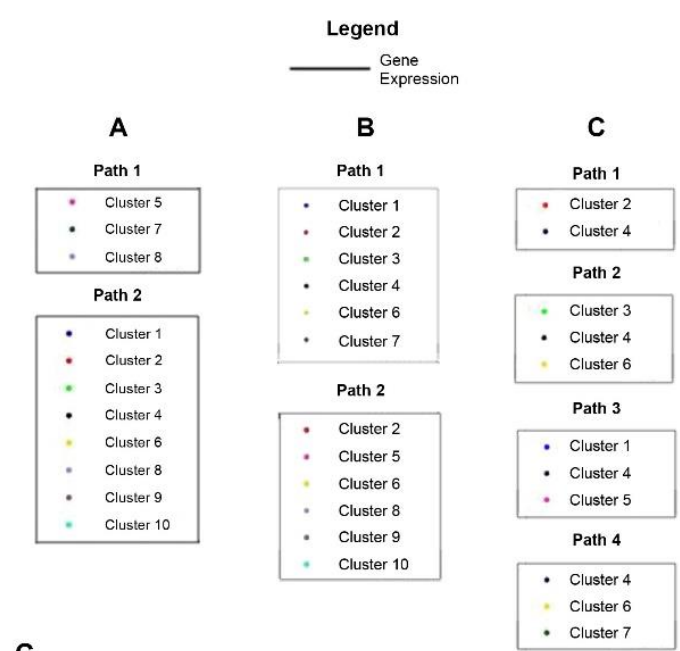

C

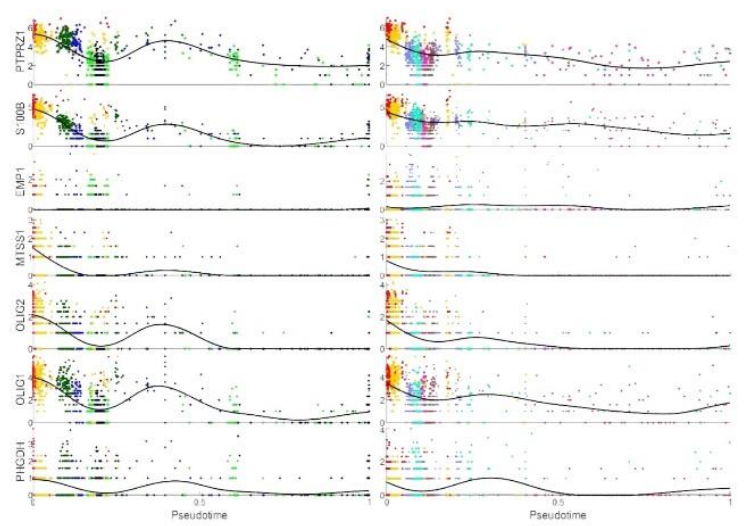

FIGURE 3. Reconstructing pseudotime dynamics in GBM/GSC cell fate decisions of the Waddington landscape. Average normalized gene expression in cells plotted along pseudotime after fitting with a cubic smoothing spline (black line). Cells are colored according to cell clusters defined by scEpath. The expression patterns of the top genes identified by scEpath and BigSCale algorithms (via correlation metrics) showed significant changes along the pseudotime trajectory inferred by scEpath algorithm. Selected gene markers in A) pediatric GBM, B) adult GBM, C) adult GSC. configurations driving GBM/GSC cell fate transitions.

419 We next reverse engineered the signaling networks coordinating the information flow in GBM

420 and GSC using Partial Information Decomposition and Context (PIDC). Though the network

421 topography may seem similar, the arrangement of the interactions from highest influence on the 422 information flow (i.e., top PID scores) to those of the weakest interactions (lowest PID scores) 
423 vary for each patient group. As seen in Figure 4A, OLIG1 and OLIG2 have the highest PID

424 score of 1.9508 , followed by S100B and PTPRZ1 interaction with a PID score of 1.9303 in

425 pediatric GBM, suggesting a strong relationship between these two genes in the complex

426 network steering their cell fate decisions (Fig 4A). We found that S100A10 and EMP1 have the

427 highest interaction in adult GBM with a PID score of 1.9517 (Fig 4B), whereas NACA and TPT1

428 had the highest interaction in adult GSC with a PID score of 1.9628 (Fig 4C). A distinct pattern

429 was observed in the PIDC regulatory network of adult GSC sample BT127 (highest quality GSC

430 cells). The highest interaction was observed between PHGDH and TIMELESS at a PID score of

431 2.762. Other top interactions identified for the TF networks (Fig 4E-G) had similar

432 pseudotemporal expression dynamics (Figure S4 A-C in the Supplementary Information). ATF3

433 and DDIT3 were the top interaction markers from the critical TFs identified for pediatric GBM

434 with a PID score of 1.971 (Fig 4E). EGR1 and FOSB in the adult GBM group (Fig 4F), and

435 YBX1 and HMGB1 were identified as the top interaction TF markers, with PID score of 1.992

436 (Fig 4G). These results suggest the reconfiguration of the nodes within the same complex

437 signaling network may characterize GSC cells from GBM cells and distinguish pediatric GBM

438 from adult GBM cell fate dynamics. 

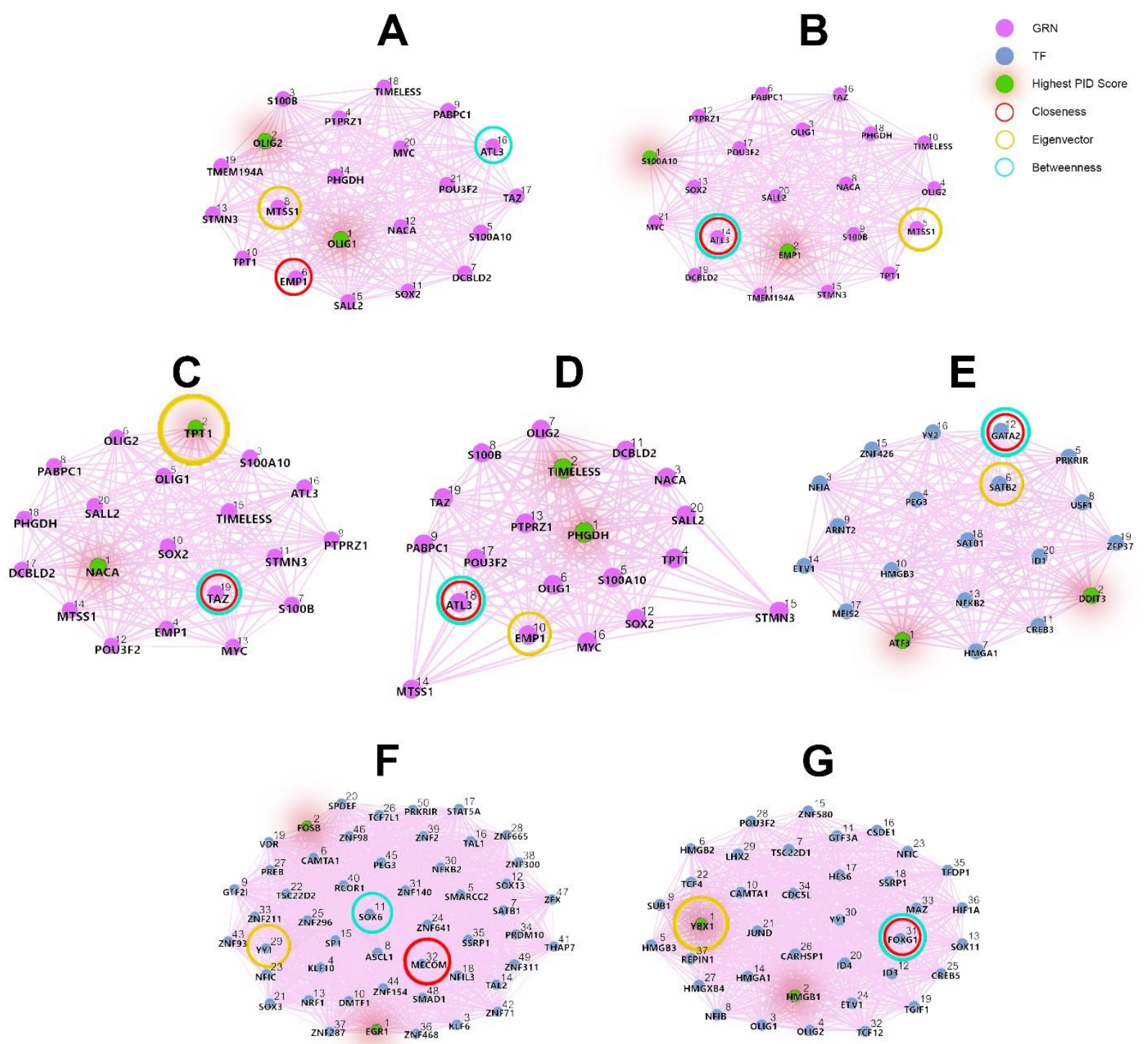

FIGURE 4. Mathematical modelling identifies key regulatory genes driving GBM GSC sample BT127, E) pediatric GBM transcription factors, F) adult GBM transcription factors, and $\mathrm{G}$ ) adult GSC transcription factors. In each, the signaling networks show the information flow between critical signals required for the complex cell fate dynamics. The GRN networks identified by Seurat and BigSCale are colored in violet nodes (Fig 4A-D) while the scEpath TF networks are colored in teal (Fig 4E-G). The ranks were assigned a priority index by the PID content as indicated by the numbers on the nodes. A high PID content implies a high mutual information (dependence) of those gene interactions in the information flow network. The number index on the nodes of the network correspond to the PID score in a decreasing order, where rank 1 denotes the top (highest) value. As shown in the legend, the nodes with the rings are used to identify the nodes of the networks with the highest network centrality measures as identified in Figure 5. 
3.4. Network centrality measures identify master regulators of information flow across

456 Centrality is a key property of complex networks that influences the network dynamics and

457 information flow (lacono et al., 2019). The nodes (genes or TFs) with the highest centrality in the

458 regulatory networks are the most biologically important signals. By measuring network

459 centrality, we identified the primary genes regulating communication flow across each of the

460 pediatric and adult GBM, and adult GSC networks (Table 2). In particular, we calculated the

461 global clustering coefficient that measures the total number of closed triangles (link density) in a

462 network. A clustering coefficient at its maximal value of 1 indicates that the neighbors of the

463 gene (node) $i$ form a complete graph (i.e., they all connect to each other) versus the converse

464 for a clustering coefficient of 0 (Barabási and Posfai, 2016). We observed a lower clustering

465 coefficient of 0.94 for the BT127 network in Figure 4D. In the transcription factor networks

466 reconstructed from the scEpath heatmaps (italic columns, Table 2), the GSC TF network had

467 the highest diameter, while the GBM networks (both pediatric and adult) had smaller diameters.

468 The diameter is relatively in the same order of magnitude for the PIDC networks reconstructed

469 from the Seurat-BigSCale markers (bold columns, Table 2) as they correspond essentially to the

470 same set of genes interactions. The degree of centrality of all networks in Figure 4 was 1.0 at all

471 nodes, except for the BT127 PIDC network which had a degree centrality of value of 1.0 only at

472 nodes $1,5,10,12,13$, and 16 , and a clustering coefficient of value 0.96 . The degree centrality

473 of nodes 2,7 , and 8 were 0.89 , the degree centrality of nodes 14 and 15 were roughly 0.5 , and

474 the remaining nodes had a degree centrality of 0.95 .

475

476 The closeness centrality identified genes/TFs occupying a central position in a network (lacono

477 et al., 2019). The nodes corresponding to the highest closeness centrality for each GRN

478 network were found to be Node 6 (EMP1) for pediatric GBM, Node 14 (ATL3) for adult GBM, 
479 Node 18 (ATL3) for GSC BT127, and Node 19 (TAZ) for GSC with closeness values of 1.398,

$4801.361,1.006$, and 1.184, respectively (Fig 5A).

481

482 Nodes corresponding to the maximal closeness in the pediatric GBM, adult GBM, and adult

483 GSC TF networks were found to be node 12 (GATA2), node 32 (MECOM), and node 31

484 (FOXG1), respectively with closeness measures of 1.761, 2.563, and 1.478 respectively (Fig

485 5B).

486 Betweenness centrality indicates the presence of regulatory bottlenecks (lacono et al., 2019;

487 Latora et al., 2017; Rodrigues, 2019). In our analyses, the highest betweenness measures for

488 the pediatric GBM, adult GBM, BT127 adult GSC, and adult GSC GRN networks were node 16

489 (ATL3), node 14 (ATL3), node 18 (ATL3), and node 19 (TAZ), respectively with betweenness

490 values of $0.3947,0.5842,0.2690$, and 0.4678 , respectively (Fig $5 \mathrm{C}$ ). The trends in maximal

491 betweenness values (Fig 5C) were in good agreement with the nodes contributing to the

492 maximal closeness values discussed in Fig 5A, indicating that identified nodes are critical

493 targets governing the information flow in these complex networks. The highest betweenness

494 values for the TF networks were found to be to node 12 (GATA2) for pediatric GBM, node 11

495 (SOX6) for adult GBM, and node 31 (FOXG1) for adult GSC, with values of 0.3801, 0.2279, and

4960.1539 , respectively (Fig 5D). The highest values of eigenvector centrality, a measure of

497 information flow across the network, for the GRNs were found to be node 8 (MTSS1) for

498 pediatric GBM, node 5 (MTSS1) for adult GBM, node 10 (EMP1) for BT127, and node 2 (TPT1)

499 for GSC, with measures of $0.2796,0.2827,0.2909$, and 0.2805 , respectively.

500

501 The maximal eigenvector is a measure of the hub-score, i.e., the highest authority node of hub

502 networks (Latora et al., 2017; Rodrigues, 2019). The maximal eigenvector centrality of the TF

503 networks was found to be node 6 (SATB2) for pediatric GBM, and node 29 (YY1) for adult GBM, 
504 and node 1 (YBX1) for GSC, with values of $0.2594,0.1874$, and 0.2322 , respectively. SATB2 is

505 a nuclear matrix-associated protein involved in chromatin remodelling and transcription

506 regulation during neuronal differentiation (Gyorgy et al., 2008). Interestingly, all transition genes

507 with high centrality measures identified in our network analyses, including EMP1, MTSS1,

508 ATL3, and TPT1 have a TF-binding site for YY1 (Stelzer et al., 2016; GeneCards, 2021) (see

509 Table 3).

510

511 We also performed fractal analysis on the attractors (cell clustering patterns) in the scEpath

512 Waddington landscapes. The fractal dimension scores obtained on the cell state attractors on

513 the energy landscape were compared across all groups (pediatric GBM $(n=7)$, adult GBM $(n=$

514 18), and GSC $(n=28))$. The mean fractal dimension scores of the pediatric $G B M$, adult GBM,

515 and adult GSC groups were $1.502 \pm 0.099,1.509 \pm 0.091$, and $1.588 \pm 0.051$, respectively

516 (Figure 5G). The FD scores of the two GBM groups were nearly identical, while a statistically

517 significant difference was observed from the GSC group. The multifractal spectrum $f(\alpha)$ was

518 extracted from the multifractal spectra of the individual cancer samples energy landscape

$519(\mathrm{n}=54)(\mathrm{Fig} 5 \mathrm{H})$. Only the difference between GSC versus adult GBM was found to be

520 statistically significant $(p=0.0201)$ by a Kolmogorov-Smirnov test. The pediatric GBM, and adult

521 GBM and GSC groups had a maximal multifractal spectrum $f(\alpha)$ value of $1.499 \pm 0.092,1.462$

$522 \pm 0.066$, and $1.521 \pm 0.075$, respectively.

\begin{tabular}{|c|l|l|l|l|l|l|l|}
\hline $\begin{array}{l}\text { Network } \\
\text { Properties }\end{array}$ & $\begin{array}{l}\text { Pediatric } \\
\text { GBM }\end{array}$ & $\begin{array}{l}\text { Adult } \\
\text { GBM }\end{array}$ & $\begin{array}{l}\text { Adult } \\
\text { GSC }\end{array}$ & $\begin{array}{l}\text { BT127 } \\
\text { (Adult } \\
\text { GSC) }\end{array}$ & $\begin{array}{l}\text { Pediatric } \\
\text { GBM } \\
\text { (TF } \\
\text { Network) }\end{array}$ & $\begin{array}{l}\text { Adult } \\
\text { GBM } \\
\text { (TF } \\
\text { Network) }\end{array}$ & $\begin{array}{l}\text { Adult } \\
\text { GSC (TF } \\
\text { Network) }\end{array}$ \\
\hline$G(V, E)$ & $(21,210)$ & $(21,210)$ & $(20,190)$ & $(20,174)$ & $(20,190)$ & $\begin{array}{l}(50, \\
1225)\end{array}$ & $(37,666)$ \\
\hline Center & 16 & 14 & 19 & 18 & 8 & 15 & 31 \\
\hline Diameter & 3.037 & 3.283 & 3.490 & 3.106 & 1.869 & 1.474 & 3.936 \\
\hline $\begin{array}{l}\text { Global } \\
\text { Clustering } \\
\text { Coefficient }\end{array}$ & 1.00 & 1.00 & 1.00 & 0.94 & 1.00 & 1.00 & 1.00 \\
\hline
\end{tabular}


TABLE 2. General Properties of Inferred Complex Networks. $G(V, E)$ denotes the graph with the number of vertices $V$ (the genes) and number of edges $E$ for each inferred GRN network. The center values designate to the node index (gene) acting as the center of the simple weighted network. The clustering coefficient captures the degree to which the neighbors of a given node link to each other.

A

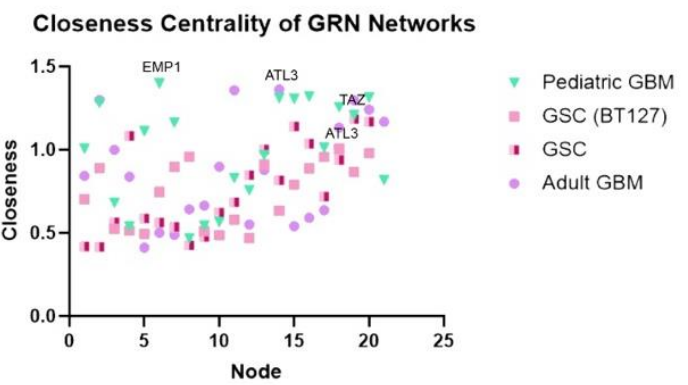

C

Betweenness Centrality of GRN Networks

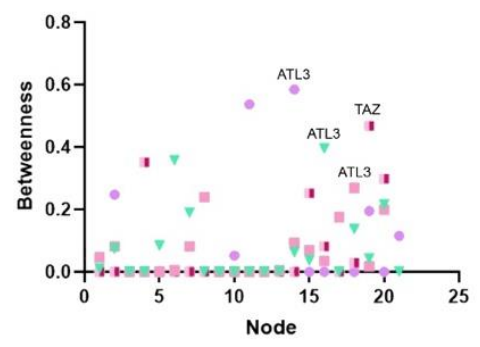

E

Eigenvector Centrality of GRN Networks

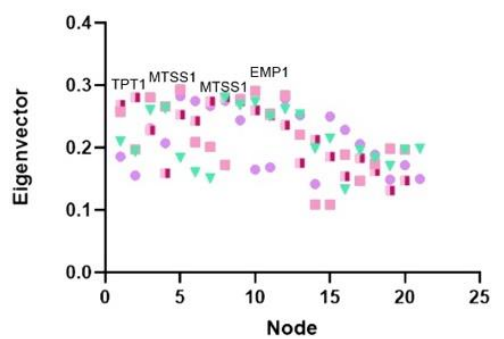

G

Boxcount Fractal Dimension

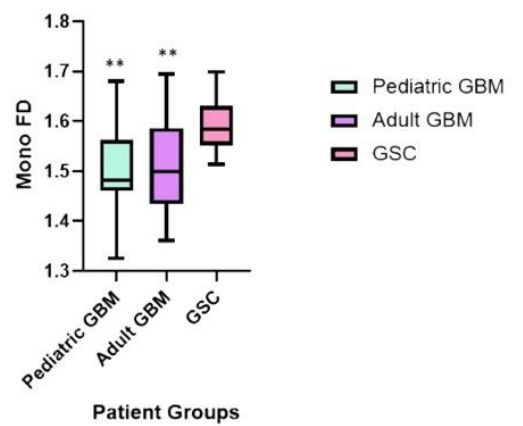

B

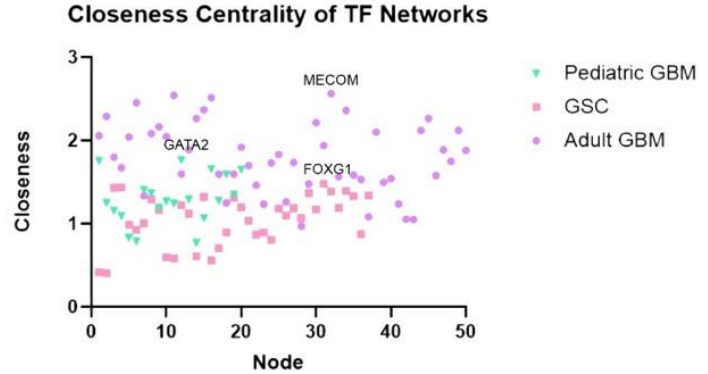

D

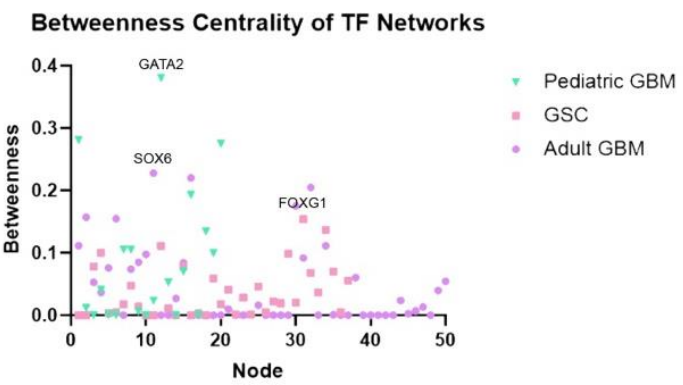

$\mathbf{F}$

7 Pediatric GBM

- GSC_BT127

- GSC

- Adult GBM

Eigenvector Centrality of TF Networks

Pediatric GBM

GSC

- Adult GBM

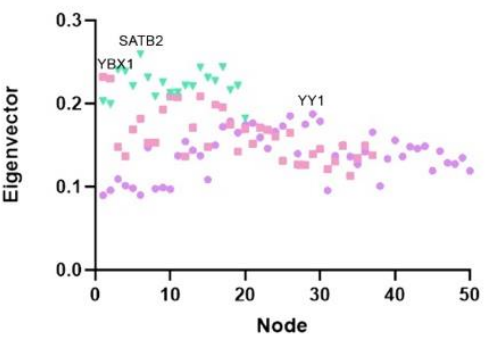

H

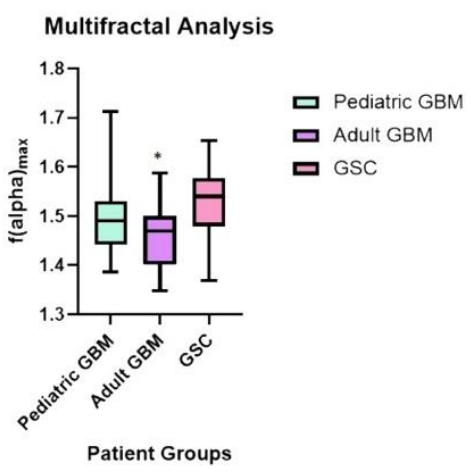



networks. Three network centrality measures are assessed on the reconstructed GBM/GSC networks. Three different network centralities were computed on the reconstructed networks: closeness, betweenness, and eigenvector centrality. The genes (nodes) occupying the highest of these centrality measures correspond to critical nodes steering the information flow in the complex signaling networks governing GBM/GSC cell fate transition dynamics. A) Closeness centrality of inferred GRNs. B) Closeness centrality of TF networks. C) Betweenness centrality of gene regulatory networks. D) Betweenness centrality of transcription factor networks. E) Eigenvector centrality of gene regulatory networks. F) Eigenvector centrality of transcription factor networks. G) Fractal dimension of cell state attractors on scEpath energy landscapes. A $p$-value of 0.0031 between the adult GSC and adult GBM, and $p=0.0011$ between adult GSC and pediatric GBM was calculated for the box-count algorithm's fractal dimension scores using the Kolmogorov-Smirnov test. Multifractal analysis of cell fate attractors on scEpath Waddington landscapes.

\section{DISCUSSION}

547 RNA-seq data from pediatric and adult GBM, and adult GSCs to distinguish genes regulating

548 communication within these cellular populations. Our findings demonstrate the application of

549 these tools for deciphering GBM/GSC signaling networks to understand how network

550 configuration orchestrates information flow and determines cell fate dynamics.

552 Multiple clustering algorithms were deployed to cross-validate their findings and ensure that the

553 differential markers extracted for network analysis were robust, complementary, and of high

554 importance in cell fate transition/differentiation mapping. There is a high degree of heterogeneity

555 displayed by GBM stem cells. The complementarity of our results in our independent and

556 orthogonal approaches are outlined in Table 3 by the associations identified between the

557 transition genes and the scEpath TFs. Our approach using distinct clustering techniques and

558 verifying their matching or complementary results was deployed to minimize the effects of

559 expression heterogeneity and validate our findings (Krieger et al., 2020).

\begin{tabular}{|l|l|}
\hline TRANSITION GENES & TRANSCRIPTION FACTORS \\
\hline ATL3 & $\begin{array}{l}\text { YY1, FOSB, SOX6, GATA2, ATF3, EGR1, } \\
\text { MYC }\end{array}$ \\
\hline MTSS1 & YY1, ATF3, MYC \\
\hline
\end{tabular}




\begin{tabular}{|l|l|}
\hline EMP1 & YY1, FOSB, GATA2, ATF3, MYC \\
\hline TPT1 & YY1, ATF3, FOSB, SOX6, EGR1, OLIG1/2 \\
\hline PTPRZ1 & YY1, YY2, EGR1, NANOG, POUF51 \\
\hline S100B & YY1, GATA2, EGR1, SOX6, MYC \\
\hline
\end{tabular}

TABLE 3. Interactions between transition genes and transcription factors identified in

network analysis. Amidst the critical transition genes listed, the first four were identified as the central regulators of information flow across the GBM/GSC regulatory networks, while PTPRZ1 and S100B were other differential markers identified in our analyses. The list is not inclusive of all possible gene-TF interactions but restricted to the analysis of only the high importance (i.e., highest network centrality measures) scEpath TFs identified in our findings. The TF-gene interactions were identified using the GeneCards human gene database (GeneCards, 2021).

Using scEpath, we identified three and four meta-clusters in the pediatric GBM (Fig 2A) and adult GBM (Fig 2B), respectively, while sub-clusters within each meta-cluster indicated the presence of phenotypic heterogeneity and plasticity. However, the number of meta-clusters was ambiguous in the adult GSC landscape (Fig 2C), as shown by the continuous progression from the higher energy state clusters (stem-like fates) to the lower energy states indicating the potential presence of a complex attractor. An alternative measure to assess the significance of the scEpath clustering is the transition paths (cell fate trajectories). We predicted that the number of clusters identified in the pediatric GBM group corresponds to the neuronal, astrocyticmesenchymal, and oligodendrocytic lineages, mirroring the healthy brain's neurodevelopmental

577 hierarchy (Jessa et al., 2019; Couturier et al., 2020). Similarly, the four clusters identified in the 578 adult GBM group correspond to the four groups identified by Neftel et al. (2019), namely the 579 OPC-like (oligodendrocytic progenitor cell), NPC-like (neuronal progenitor cell-like), AC-like

580 (astrocytic cell-like), and MES-like (mesenchymal cell) lineages. Further, the infiltrated immune

581 cells (i.e., T-cells and macrophages) grouped into the MES-like state (Neftel et al., 2019).

582 Pediatric GBM cells showed less differentiation than the adult GBM samples, as indicated by

583 the higher energy cell-states, suggesting a closer resemblance to the GSC sample. The two cell

584 fate trajectories observed in the adult GSC sample may correspond to the transcriptional

585 gradient of two cellular states observed in the original study by Richards et al. (Fig 2C), which 
were shown to mirror normal neurodevelopment and inflammatory wound responses (Richards

587 et al., 2021).

589 The cell fate trajectories along the scEpath Waddington landscape (Figure 2A-C) were

590 determined by the transition probabilities of the probabilistic directed graph reconstructed from

591 the cell fate clusters, where the weighted edges of the networks correspond to the average

592 normalized gene expression (see Supplementary Information for additional details). scEpath

593 used the minimum directed spanning tree to find the maximum probability flow and minimal

594 number of edges along the network, since cell fates transition to lower energy states during

595 differentiation. The resulting tree approximates the cell state transition network and infers the

596 observed developmental trajectories/lineage structures. The weighted edges of the cell state

597 transition network were found to be proportional to the gene expression values seen in Figure 3,

598 where the number of developmental trajectories inferred are indicated by the path numbers in

599 Figure 2. Thus, two cell fate trajectories were detected in the pediatric GBM and adult GSC

600 samples while four developmental trajectories were observed in adult GBM.

602 In pediatric GBM, the expression of transcription factors in pseudotime was shown to be highly

603 nonlinear. Certain genes, including GATA2, were even found to be oscillatory in one trajectory

604 while demonstrating an increasing or decreasing gradient of expression along the other cell fate

605 trajectory. Likewise, patterns of other critical transition genes (TFs) were identified along the

606 attractor dynamics between the distinct transcriptional states of adult GBM and adult GSC cells.

607 Further, we found that genes such as EMP1, MTSS1, PTPRZ1 and S100B exhibited distinct

608 gene expression oscillations in one differentiation trajectory (path) over the other(s) (Figure 3).

609 These genes were also found to have TF-binding sites for the scEpath identified TFs with the

610 highest network centrality measures in our downstream analysis (Figure 4). Together, these

611 findings are indicative of a highly interconnected network of gene-TF interactions governing 
612 GBM/GSC cell fate decisions, and further suggest that the information flow across the inferred

613 networks may steer cell fate decisions towards complex attractors on the GBM/GSC

614 Waddington landscape.

616 Using network centrality measures, we identified OLIG1/2, TAZ, GATA2, FOXG1, SOX6,

617 SATB2, YY1, and gene targets ATL3, MTSS1, EMP1, and TPT1 as critical genes governing the

618 cell fate dynamics of GBM and GSC cells (Fig 5A-F). Many of these signals are

619 neurodevelopmental transcription factors involved in healthy brain development, essential for

620 conferring and maintaining cancer stem cells (GSCs). Maximal centrality scores indicated that

621 they are key regulators of the network information flow in both GBM groups and GSCs. The

622 functional significance of these transcription factors (see Supplementary Information) suggests

623 their critical role in stem cell decision-making and differentiation dynamics. Our findings indicate

624 that these genes may be strong candidates for therapeutic interventions points for the treatment

625 of GBM. Other signaling interactions such as PTPRZ1 and S100B were identified in our

626 analyses as potent clinically druggable targets in the treatment of GBM. Furthermore, we

627 predicted that GATA2 and MTSS1 may provide a common ground for interlinking

628 leukemogenesis, the complex signaling dynamics of leukemia/lymphoma affecting children, and

629 pediatric glioma/GBM (Menendez-Gonzalez et al., 2019, Schemionek et al., 2015).

631 BDM was used to distinguish which of the differential markers can accurately

632 classify/differentiate the three patient group samples (see Supplementary Information). We

633 identified FOSB, HMGB1 and EGR1 as differential signatures which can accurately predict the

634 patient groups in our single-cell analyses (see SI). The algorithmic complexity measured by the

635 BDM allowed for the identification of critical network genes differentiating GBM and GSC

636 phenotypes with the minimal information. The rationale for using gene/TF markers' BDM as a

637 phenotypic discriminant is that the algorithmic complexity denotes the shortest algorithm or 
638 minimal set of information within the complex networks inferred required to classify the distinct

639 patient groups. As such, the identified genes/TFs may be useful biomarkers for prognostic

640 screening and disease phenotyping in clinical medicine.

642 From the transcription factor (TF) networks identified by scEpath (Table 3), we distinguished

643 some TFs to form interactions with some of the differential gene markers, suggesting cellular

644 reprogramming targets for controlling GBM cell fate dynamics. Our study therefore quantifies

645 how these markers' expression vary in the cell fate transitions from stem-like to mature

646 phenotypes. For a discussion on the biological significance of key genes and transcription

647 factors identified in our analyses, see the Supplementary Results in the Supplementary

648 Information.

650 The cell fate transition markers identified in our study, including PTPRZ1, EMP1, S100B, and

651 MTSS1, are in good agreement with the findings from the original studies (SCP393 and

652 SCP503). Although some of the signatures we identified overlap with the differential expression

653 patterns of the original studies, they did not compare the co-expression of these markers

654 between GSC and GBM. Markers differentiating distinct cellular states have been previously

655 investigated (for instance, the original study by Neftel et al. identified copy number

656 amplifications of the CDK4, EGFR, and PDGFRA loci and mutations of the NF1 locus, each

657 favoring one of the four GBM phenotypes (Neftel et al., 2019)). Our study instead analyzed the

658 expression patterns which fluctuate or form a differentiation gradient across the distinct cell

659 states. Further, while previous studies have associated the differentiation markers of GBM

660 progression identified here, our study demonstrates their novel integrated application to

661 elucidate the roles of these network biomarkers in GBM cell fate decisions and differentiation

662 dynamics. Indeed, while many of the identified genes or TFs have been previously studied in

663 the context of neurodevelopmental regulation and glioma cell fate dynamics, most of those 
664 selected in our analyses are not yet documented in glioblastoma cell fate control. As such, we

665 propose the identified interactions in Table 3 may provide clinically relevant GBM-specific

666 precision therapeutics, and that our network analyses provide a quantitative tool to characterize

667 which of the markers were of high importance (i.e., high centrality measures) in cell fate control,

668 plasticity regulation, and transition dynamics. Future studies should exploit tools from

669 algorithmic complexity theory including algorithmic network perturbation analysis (i.e., quantify

670 the BDM changes across a network by node or link deletion) to better elucidate the inferred

671 network dynamics in cancer cell fate control and regulation.

673 While previous GBM gene regulatory network inference methods vary from our approaches, our

674 findings are consistent with their results. For example, Sun et al. found 15 hub genes in GBM-

675 specific miRNA-TF networks, including PDGFRA and SOX11, and 6 hub TFs (including GATA1)

676 as key regulators of GBM dynamics (Sun et al., 2012). In our study, we also identified PDGFRA

677 and SOX11 as hub genes of the inferred GBM networks, and found that GATA2, an alternate

678 isoform, overlapped with these findings. However, Sun et al. (2012) did not compare GBM of

679 different age groups nor consider GBM-derived stem cells for reconstruct their differentiation

680 networks. Similarly, a network inference study by Ping et al. (2015) revealed 17 hub genes in

681 GBM networks, including EGFR and PDGFRA, as gene signatures of the proneural GBM

682 subtype, both of which were identified in our analyses. In another study, GSEA and IPA-based

683 gene enrichment pathway analysis discovered TAZ as a key regulator of GBM networks

684 (Bozdag et al., 2014), which was also identified as a master regulator of GBM differentiation

685 dynamics in our analyses.

687 Using multi-omic analyses, Suva et al. distinguished OLIG2, POU3F2 SALL2, and SOX2 as hub 688 genes of GBM stemness networks critical for their tumor-propagation potential (Suvà et al., 689 2014). Our findings identified OLIG2 as a master control gene of GBM differentiation dynamics 
690 and established a connection between SOX2 expression and the critical hub gene FOXG1.

691 Further, some epigenetic profiling studies have shown that aberrant histone modifications and

692 methylation profiles are molecular signatures driving pediatric GBM and distinguishing them

693 from their adult counterparts (Jones et al., 2017, Lulla et al., 2016, Sturm et al., 2012). Sturm et

694 al. (2012) revealed that the TFs OLIG1, OLIG2 and FOXG1 are the master regulators of the hub

695 gene networks driving these oncohistone pediatric GBM variants (i.e., K27M and G34V/R).

696 Similar findings were recently reported by Wang et al. (2021), who identified the same set of

697 TFs as critical drivers of pediatric high-grade gliomas' epigenetic landscapes. We identified all

698 three TFs reported by Sturm et al. and Wang et al. in our network approaches as critical

699 regulators of GBM cell fate dynamics. Thus, our findings recapitulate the complex network

700 dynamics driving the oncohistone variants of pediatric GBM and validate and extend previous

701 findings.

702

703 It should be noted that there is a good deal of heterogeneity within the single-cell datasets

704 across and with the patient groups. The original datasets contained 8 pediatric GBM samples,

70520 adult GBM samples, and 28 adult GSC samples. For the initial clustering (i.e., differential

706 discovery using Seurat and BigSCale), samples--two adult GBM and one pediatric GBM-- with

707 the highest drop-out rate (i.e., zero counts) were removed as a data filtering and quality control

708 step prior to normalization. Subsequently, the number of adult GBM samples in the scEpath

709 analysis was randomly selected to closely match the cell count numbers of the adult GSC

710 patient groups. The down-sampling of GSC samples was necessary since scEpath analysis has

711 a computational limitation on the number of samples which can be processed (roughly 2500

712 cells). As noted in the Methods, selecting a different combination of GSC samples did not

713 change the results and including the removed samples did not change the differential marker

714 discovery or expression analyses. Indeed, the global clustering patterns remained the same

715 although there was greater dispersion in the local sub-clusters in the Seurat and BigSCale 
716 pattern space. However, including all $n=8$ pediatric GBM patient samples generated a shorter

717 list of transition genes with abrupt transitions between the distinct phenotypes.

719 A limitation of our study is that we did not have access to pediatric GSC cells, given that adult

720 GSC data have only recently been described (Richards et al., 2021). There may be other hidden

721 causal interactions interconnecting the nodes of the complex networks we inferred that were not

722 identified due to lack of data. Further, the lack of time-series scRNA-Seq counts is a barrier to

723 understanding the complex dynamics of GBM/GSC networks. The pseudotemporal dynamics

724 consist of inferred cell fate trajectories in a dimensionality-reduced data space (i.e., PCA space)

725 by transcriptional similarity of cell fates. Ribosomal proteins and certain cytoskeletal markers

726 (housekeeping genes) were also not pooled with the differential expression signatures for

727 network inference (Figure S1).

729 This proof-of-concept study provides a comprehensive method to dissect the cybernetics of

730 cancer cellular ecosystems and their cell fate dynamics. Current bioinformatic pipelines in

731 cancer data science largely fail to reconcile the complex dynamics and temporal features of

732 GBM transcriptional states, as they either take a reductionist approach to inferring gene

733 expression patterns or rely on statistical correlation methods. In contrast, our framework

734 provides a pipeline for causal pattern discovery and thereby allows the prediction/forecasting of

735 how the differentially expressed transition genes control and regulate cell fate decision-making.

736 Further, our approach allows for the mapping of these cancer cell fate behaviors to information

737 flow across the inferred complex networks. Thus, these causal inference tools shed light on

738 emergent behaviors in cell fate decisions such as transcriptional heterogeneity from a dynamical

739 systems perspective. As such, we propose our methodological framework may provide a

740 complementary and potentially more useful means to assess how the heterogeneous cancer 
741 phenotypes exhibit adaptive (emergent) behaviors and help forecast their dynamic response to

742 drug/therapeutic perturbations at the level of molecular interactions.

743

\section{CONCLUSION}

745 This study demonstrates the use of complex systems approaches in deciphering the cybernetics

746 of GBM/GSC networks, and shows how signaling dynamics differ between pediatric GBM, adult

747 GBM, and adult GSC populations. By identifying transcription factors and genes, our combined

748 approach serves as one part of the precision medicine toolbox for the treatment of GBM,

749 suggesting both precision therapeutic targets and GBM reprogramming factors.

751 Prospective studies should explore the use of artificial neural networks, including Deep Learning

752 algorithms, for single-cell transcriptomic analyses. Further, causal inference-based network

753 inference methods such as Bayesian networks and algorithmic information dynamics should be

754 investigated for GBM regulatory networks reconstruction. The epigenetic regulation of our

755 identified transcriptional networks must be explored using high-throughput multi-omics datasets.

756 Our network approaches should be extended to protein-protein interaction networks, epigenetic

757 networks, and metabolic networks to investigate multi-omic levels of GBM heterogeneity,

758 including oncohistone variants (i.e., K27M, K36M, G34V/R) and IDH1/2-mutants observed in

759 pediatric gliomas (GBM).

760 DECLARATIONS

761 DECLARATIONS STATEMENT: The authors declare no competing interests.

762 ETHICS APPROVAL AND CONSENT TO PARTICIPATE: Not Applicable

763 AUTHOR CONTRIBUTIONS: AU performed the algorithms, wrote, and edited the manuscript.

764 MC supervised, wrote, and edited the manuscript.

765 FUNDING: MC was funded by Natural Science and Engineering Research Council of Canada 766 Discovery Grant RGPIN-2018-04546 and an FRQS Research Scholar grant (J1). 
768 The datasets supporting the conclusions of this article are available in the Single Cell Portal

769 repository:

770 https://singlecell.broadinstitute.org/single cell/study/SCP393/single-cell-rna-seq-of-adult-and-

771 pediatric-glioblastoma\#study-summary

772

773

https://singlecell.broadinstitute.org/single cell/study/SCP503/gradient-of-developmental-and-

774 injury-reponse-transcriptional-states-define-functional-vulnerabilities-underpinning-glioblastoma-

heterogeneity\#study-download

777 All Codes and Algorithms used for the single-cell data analysis are available in the project

778 GitHub page:

779 https://github.com/Abicumaran/GBM Complexity I

781 Software/Algorithms:

782 Seurat

783 Project name: Seurat V3

784 Project home page: https://github.com/satijalab/seurat/

785 Archived version: 10.1016/j.cell.2019.05.031

786 Operating system(s): Platform independent

787 Programming language: $R$

788 Other requirements: Not Applicable

789 License: GNU Public License (GPL 3.0)

790 Any restrictions to use by non-academics: Not Applicable

791

792

793

794

795

796

797

798

799

800

801

802

803

804

805

806

807

808

809

\section{BigScale}

Project name: BigScale V2

Project home page: https://github.com/iaconogi/BigSCale2

Archived version: 10.1186/s13059-019-1713-4

Operating system(s): Platform independent

Programming language: $\mathrm{R}$

Other requirements: $\mathrm{C}_{++}$

License: Not Applicable

Any restrictions to use by non-academics: Not Applicable

\section{scEpath}

Project name: single-cell Energy path (scEpath)

Project home page: https://github.com/sqiin/scEpath

Archived version: 10.1093/bioinformatics/bty058

Operating system(s): Platform independent

Programming language: MATLAB

Other requirements: $\mathrm{C}_{++}$

License: Not Applicable

Any restrictions to use by non-academics: Not Applicable

$\underline{\text { OACC }}$ 
814 Project name: Online Algorithmic Complexity Calculator V3

815 Project home page: https://github.com/algorithmicnaturelab/OACC

816 Archived version: 10.1016/j.isci.2019.07.043

817 Operating system(s): Platform independent

818 Programming language: $R$

819 Other requirements: Not Applicable

820 License: GNU Public License (GPL 3.0)

821 Any restrictions to use by non-academics: Not Applicable

\section{Network Inference}

Project name: NetworkInference.jl and Partial Information Decomposition (PID)

Project home page: https://github.com/Tchanders/NetworkInference.jl

Archived version: 10.1016/j.cels.2017.08.014

Operating system(s): Platform independent

Programming language: Julia

Other requirements: Not Applicable

License: MIT "Expat" License

833

Any restrictions to use by non-academics: Not Applicable

\section{Julia LightGraphs}

Project name: LightGraphs.jl V1.3

Project home page: https://github.com/JuliaGraphs/SimpleWeightedGraphs.jl

Archived version: Not Applicable

Operating system(s): Platform independent

Programming language: Julia

Other requirements: Jupyter Notebook and HTML

License: MIT "Expat" License

Any restrictions to use by non-academics: Not Applicable

\section{SciKit-learn:}

Project name: Scikit-learn

Project home page: https://scikit-learn.org/ or https://github.com/scikit-learn/scikit-learn

Archived version: http://jmlr.org/papers/v12/pedregosa11a.html

Operating system(s): Platform independent

Programming language: Python ( $\geq$ V3.7)

Other requirements: NumPy $(\geq 1.14 .6)$, SciPy $(\geq 1.1 .0)$, joblib $(\geq 0.11)$, threadpoolctl $(\geq 2.0 .0)$, Google Colab or Jupyter Notebook

License: 3-Clause BSD license

Any restrictions to use by non-academics: Not Applicable

858 Project home page: https://imagej.nih.gov/ij/plugins/fraclac

859 Archived version: Not Applicable

860 Operating system(s): Platform independent

861 Programming language: Java

862 Other requirements: Not Applicable

863 License: National Institute of Health (NIH) Public License

864 Any restrictions to use by non-academics: Not Applicable 


\section{REFERENCES}

867

868

869

870

871

872

873

874

875

876

877

878

879

880

881

882

883

884

1. Alifieris, C., \& Trafalis, D. T. Glioblastoma multiforme: Pathogenesis and treatment. Pharmacology \& therapeutics, 152, 63-82. (2015). https://doi.org/10.1016/i.pharmthera.2015.05.005

2. Jung, E., Alfonso, J., Osswald, M., Monyer, H., Wick, W., and Winkler, F. Emerging intersections between neuroscience and glioma biology. Nat. Neurosci. 22, 1951-1960. (2019). https://doi.org/10.1038/s41593-019-0540-y.

3. Suvà, M. L., Rheinbay, E., Gillespie, S. M., Patel, A. P., Wakimoto, H., Rabkin, S. D., Riggi, N., Chi, A. S., Cahill, D. P., Nahed, B. V., Curry, W. T., Martuza, R. L., Rivera, M. N., Rossetti, N., Kasif, S., Beik, S., Kadri, S., Tirosh, I., Wortman, I., Shalek, A. K., ... Bernstein, B. E. Reconstructing and reprogramming the tumor-propagating potential of glioblastoma stem-like cells. Cell, 157(3), 580-594. (2014). https://doi.org/10.1016/i.cell.2014.02.030

4. Jia, D., Jolly, M. K., Kulkarni, P., \& Levine, H. Phenotypic Plasticity and Cell Fate Decisions in Cancer: Insights from Dynamical Systems Theory. Cancers, 9(7), 70. (2017). https://doi.org/10.3390/cancers9070070

5. Jin, S., MacLean, A.L., Peng, T., Nie, Q. scEpath: Energy Landscape-based Inference of Transition Probabilities and Cellular Trajectories from Single-cell Transcriptomic Data. Bioinformatics 34(12):2077-2086. (2018). 10.1093/bioinformatics/bty058.

6. lacono, G., Massoni-Badosa, R. \& Heyn, H. Single-cell transcriptomics unveils gene regulatory network plasticity. Genome Biol 20: 110. (2019). 10.1186/s13059-019-1713-4.

7. Paugh, B. S., Qu, C., Jones, C., Liu, Z., Adamowicz-Brice, M., Zhang, J., et al. Integrated molecular genetic profiling of pediatric high-grade gliomas reveals key differences with the adult disease. J Clin Oncol. 28(18), 3061-3068 (2010). doi: 10.1200/JCO.2009.26.7252.

8. Jones, C., Karajannis, M. A., Jones, D., Kieran, M. W., Monje, M., Baker, S. J. et al. Pediatric high-grade glioma: biologically and clinically in need of new thinking. Neurooncology, 19(2), 153-161 (2017). doi: 10.1093/neuonc/now101.

9. Schwartzentruber, J., Korshunov, A., Liu, X.-Y., Jones, D. T. W., Pfaff, E., Jacob, K., ... Tönjes, M. Driver mutations in histone H3.3 and chromatin remodelling genes in paediatric glioblastoma. Nature, 482(7384), 226-231. (2012). doi:10.1038/nature10833

10. Sturm, D., Witt, H., Hovestadt, V., Khuong-Quang, D. A., Jones, D. T., Konermann, C., Pfaff, E., Tönjes, M., Sill, M., Bender, S., Kool, M., Zapatka, M., Becker, N., Zucknick, M., Hielscher, T., Liu, X. Y., Fontebasso, A. M., Ryzhova, M., Albrecht, S., Jacob, K., ... Pfister, S. M. Hotspot mutations in H3F3A and IDH1 define distinct epigenetic and biological subgroups of glioblastoma. Cancer cell, 22(4), 425-437. (2012). https://doi.org/10.1016/i.ccr.2012.08.024 
11. Lulla RR, Saratsis AM, Hashizume R. Mutations in chromatin machinery and pediatric high-grade glioma. Sci Adv. 2(3): e1501354. (2016). 10.1126/sciadv.1501354.

12. Harutyunyan, A. S., Krug, B., Chen, H., Papillon-Cavanagh, S., Zeinieh, M., De Jay, N., ... Majewski, J. H3K27M induces defective chromatin spread of PRC2-mediated repressive $\mathrm{H} 3 \mathrm{~K} 27 \mathrm{me} 2 / \mathrm{me} 3$ and is essential for glioma tumorigenesis. Nature Communications, 10(1262). (2019). doi:10.1038/s41467-019-09140-x

13. Xiong, S., Feng, Y., and Cheng, L. Cellular reprogramming as a therapeutic target in cancer. Trends Cell Biol. 29 (8): 623-34. (2019). 10.1016/j.tcb.2019.05.001.

14. Yabo, Y. A., Niclou, S. P., \& Golebiewska, A. (2021). Cancer cell heterogeneity and plasticity: A paradigm shift in glioblastoma. Neuro-oncology, noab269. Advance online publication. https://doi.org/10.1093/neuonc/noab269

15. Wolfram, S. "Complex Systems Theory," in Emerging Syntheses in Science: Proceedings of the Founding Workshops of the Santa Fe Institute, Santa Fe, New Mexico (D. Pines, ed.), Redwood City, CA: Addison-Wesley, pp. 183-189. (1988).

16. Shalizi, C.R. Methods and Techniques of Complex Systems Science: An Overview, Complex Systems Science in Biomedicine (T. S. Deisboeck and J. Y. Kresh, eds.), New York: Springer pp. 33-114. (2006).

17. Thurner, S., Klimek, P., and Hanel, R. Introduction to the Theory of Complex Systems (USA: Oxford University Press, 2018).

18. Huang, S., Ernberg, I., \& Kauffman, S. Cancer attractors: a systems view of tumors from a gene network dynamics and developmental perspective. Seminars in cell \& developmental biology, 20(7), 869-876. (2009). https://doi.org/10.1016/j.semcdb.2009.07.003

19. Barabási, A.-L., and Oltvai, Z.N. Network biology: understanding the cell's functional organization. Nat. Rev. Genet. 5, 101-113. (2004). https://doi.org/10.1038/nrg1272.

20. Neftel, C., Laffy, J., Filbin, M.G., Hara, T., Shore, M.E., Rahme, G.J., Richman, A.R., Silverbush, D., Shaw, M.L., Hebert, C.M. et al. An Integrative Model of Cellular States, Plasticity, and Genetics for Glioblastoma. Cell 178(4). pp. 835-849.e21. (2019). 10.1016/j.cell.2019.06.024.

21. Richards, L.M., Whitley, O.K.N., MacLeod, G., Cavalli, F.M.G., Coutinho, F.J., Jaramillo, J.E., Svergun, N., Riverin, M., Croucher, D.C., Kushida, M. et al. Gradient of Developmental and Injury Response transcriptional states defines functional vulnerabilities underpinning glioblastoma heterogeneity. Nat Cancer. 2:157-173. (2021). 10.1038/s43018-020-00154-9. 
956

957

958

959

960

961

962

963

964

965

966

967

968

969

970

971

972

973

974

975

976

977

978

979

980

981

982

983

984

985

986

987

988

989

990

991

992

993

994

995

996

997

998

999

1000

1001

1002

22. Stuart, T., Butler, A., Hoffman, P., Hafemeister, C., Papalexi, E., Mauck, W. M., 3rd, Hao, Y., Stoeckius, M., Smibert, P., \& Satija, R. Comprehensive Integration of SingleCell Data. Cell, 177(7): 1888-1902.e21. (2019). 10.1016/j.cell.2019.05.031

23. Waddington, C.H. The strategy of the Genes; a Discussion of Some Aspects of Theoretical Biology (Allen and Unwin, London. 1957)

24. Mandelbrot, B. The Fractal Geometry of Nature (San Francisco: W.H. Freeman and Company, 1982).

25. Coffey, S.D. Self organization, complexity, and chaos: the new biology for medicine. Nat. Med 4, 882-885. (1998). https://doi.org/10.3109/07357909709047603.

26. Baish, J.W., and Jain, R.K. Cancer, angiogenesis and fractals. Nat. Med. 4 (984). (1998). https://doi.org/10.1038/1952.

27. Strogatz, S.H. Nonlinear Dynamics and Chaos: With Applications to Physics, Biology, Chemistry, and Engineering (Boulder, CO: Westview Press, 2015).

28. Chan, T. E et al. Gene Regulatory Network Inference from Single-Cell Data Using Multivariate Information Measures. Cell systems vol. 5(3): 251-267.e3. (2017). doi:10.1016/j.cels.2017.08.014

29. Zenil, H., Kiani, N.A., and Tegnér, J. Methods of Information Theory and Algorithmic Complexity for Network Biology, Seminars in Cell \& Developmental Biology, 51: 32-43. (2016). doi: 10.1016/j.semcdb.2016.01.011.

30. Zenil, H., Kiani, N. A., Marabita, F., Deng, Y., Elias, S., Schmidt, A., Ball, G., \& Tegnér, J. An Algorithmic Information Calculus for Causal Discovery and Reprogramming Systems. iScience, 19: 1160-1172. (2019). 10.1016/j.isci.2019.07.043

31. Verhaak RG, Hoadley KA, Purdom E, et al. Integrated genomic analysis identifies clinically relevant subtypes of glioblastoma characterized by abnormalities in PDGFRA, IDH1, EGFR, and NF1. Cancer Cell. 17(1):98-110 (2010). doi: 10.1016/i.ccr.2009.12.020

32. Barabási, A.L., and Posfai, M. Network Science (Cambridge University Press, UK. 2016)

33. Gyorgy, A. B., Szemes, M., de Juan Romero, C., Tarabykin, V., \& Agoston, D. V. SATB2 interacts with chromatin-remodeling molecules in differentiating cortical neurons. The European journal of neuroscience, 27(4), 865-873. (2008). https://doi.org/10.1111//.1460-9568.2008.06061.x

34. Stelzer, G., Rosen R, Plaschkes I, Zimmerman S, Twik M, Fishilevich S, Iny Stein T, Nudel R, Lieder I, Mazor Y, Kaplan S, Dahary D, Warshawsky D, Guan - Golan Y, Kohn A, Rappaport N, Safran M, and Lancet D. The GeneCards Suite: From Gene Data Mining to Disease Genome Sequence Analysis, Current Protocols in Bioinformatics. 54:1.30.1 - 1.30.33. (2016). doi: 10.1002 / cpbi.5. 
1003

1004

1005

1006

1007

1008

1009

1010

1011

1012

1013

1014

1015

1016

1017

1018

1019

1020

1021

1022

1023

1024

1025

1026

1027

1028

1029

1030

1031

1032

1033

1034

1035

1036

1037

1038

1039

1040

1041

1042

1043

1044

1045

1046

1047

1048

1049

35. GeneCards: The Human Gene Database. https://www.genecards.org/ (GCID: GC12P013196; GC08M124550) (Weizmann Institute of Science; accessed on July 3, 2021)

36. Latora, V. et al., Centrality Measures. Complex Networks: Principles, Methods and Applications (Cambridge University Press, Cambridge, UK. 2017)

37. Rodrigues, F.A. Network centrality: an introduction. arXiv:1901.07901 [physics.soc-ph] (2019)

38. Krieger, M. S., Moreau, J. M., Zhang, H., Chien, M., Zehnder, J. L., \& Craig, M. A Blueprint for Identifying Phenotypes and Drug Targets in Complex Disorders with Empirical Dynamics. Patterns 1(9) 2020). 100138. https://doi.org/10.1016/i.patter.2020.100138

39. Jessa, S., Blanchet-Cohen, A., Krug, B., Vladoiu, M., Coutelier, M., Faury, D., Poreau, B., De Jay, N., Hébert, S., Monlong, J., Farmer, W. T., Donovan, L. K., Hu, Y., McConechy, M. K., Cavalli, F., Mikael, L. G., Ellezam, B., Richer, M., Allaire, A., Weil, A. G., ... Kleinman, C. L. (2019). Stalled developmental programs at the root of pediatric brain tumors. Nature genetics, 51(12), 1702-1713. https://doi.org/10.1038/s41588-019$\underline{0531-7}$

40. Couturier, C. P., Ayyadhury, S., Le, P. U., Nadaf, J., Monlong, J., Riva, G., Allache, R., Baig, S., Yan, X., Bourgey, M., Lee, C., Wang, Y., Wee Yong, V., Guiot, M. C., Najafabadi, H., Misic, B., Antel, J., Bourque, G., Ragoussis, J., \& Petrecca, K. (2020). Single-cell RNA-seq reveals that glioblastoma recapitulates a normal neurodevelopmental hierarchy. Nature communications, 11(1), 3406. https://doi.org/10.1038/s41467-020-17186-5

41. Menendez-Gonzalez, J. B., Vukovic, M., Abdelfattah, A., Saleh, L., Almotiri, A., Thomas, L. A., Agirre-Lizaso, A., Azevedo, A., Menezes, A. C., Tornillo, G., Edkins, S., Kong, K., Giles, P., Anjos-Afonso, F., Tonks, A., Boyd, A. S., Kranc, K. R., \& Rodrigues, N. P. Gata2 as a Crucial Regulator of Stem Cells in Adult Hematopoiesis and Acute Myeloid Leukemia. Stem cell reports, 13(2), 291-306. (2019). https://doi.org/10.1016/i.stemcr.2019.07.005

42. Schemionek, M., Kharabi Masouleh, B., Klaile, Y., Krug, U., Hebestreit, K., Schubert, C., ... Koschmieder, S. Identification of the Adapter Molecule MTSS1 as a Potential Oncogene-Specific Tumor Suppressor in Acute Myeloid Leukemia. PLOS ONE, 10(5), e0125783. (2015). 10.1371/journal.pone.0125783.

43. Sun, J., Gong, X., Purow, B., \& Zhao, Z. (2012). Uncovering MicroRNA and Transcription Factor Mediated Regulatory Networks in Glioblastoma. PLOS computational biology, 8(7), e1002488. https://doi.org/10.1371/journal.pcbi.1002488

44. Ping, Y., Deng, Y., Wang, L., Zhang, H., Zhang, Y., Xu, C., Zhao, H., Fan, H., Yu, F., Xiao, Y., \& Li, X. (2015). Identifying core gene modules in glioblastoma based on multilayer factor-mediated dysfunctional regulatory networks through integrating multi- 
dimensional genomic data. Nucleic acids research, 43(4), 1997-2007. https://doi.org/10.1093/nar/gkv074

45. Bozdag, S., Li, A., Baysan, M., \& Fine, H. A. (2014). Master regulators, regulatory networks, and pathways of glioblastoma subtypes. Cancer informatics, 13(Suppl 3), 3344. https://doi.org/10.4137/CIN.S14027

46. Wang, J., Huang, T. Y., Hou, Y., Bartom, E., Lu, X., Shilatifard, A., Yue, F., \& Saratsis, glioma. Science advances, 7(23), eabg4126. https://doi.org/10.1126/sciadv.abg4126

1061

1062

1063 